\title{
Geometry symmetry-free and Higher-order Optical Bound States in the Continuum
}

\section{Qingjia Zhou}

Soochow University

\section{Yangyang Fu}

Nanjing University of Aeronautics and Astronautics

\section{Lujun Huang}

University of New South Wales https://orcid.org/0000-0002-9343-796X

\section{Qiannan Wu}

North University of China

\section{Andrey Miroshnichenko}

UNSW Canberra https://orcid.org/0000-0001-9607-6621

\section{Lei Gao}

Soochow University

Yadong Xu ( $\nabla$ ydxu@suda.edu.cn)

Soochow University

\section{Article}

Keywords: photonic systems, zero-index metamaterial (ZIM), bound states in the continuum (BICs), geometrical symmetr

Posted Date: January 14th, 2021

DOl: https://doi.org/10.21203/rs.3.rs-138091/v1

License: (c) (1) This work is licensed under a Creative Commons Attribution 4.0 International License. Read Full License

Version of Record: A version of this preprint was published at Nature Communications on July 19th, 2021. See the published version at https://doi.org/10.1038/s41467-021-24686-5. 


\title{
Geometry symmetry-free and Higher-order Optical Bound States in the Continuum
}

\author{
Qingjia Zhou ${ }^{1,2}$, Yangyang $\mathrm{Fu}^{3,}{ }^{*}$, Lujun Huang ${ }^{4}$, Qiannan $\mathrm{Wu}^{5}$, Andrey Miroshnichenko ${ }^{4}$, Lei \\ $\mathrm{GaO}^{1,2, *} \&$ Yadong $\mathrm{Xu}^{1,2, *}$ \\ ${ }^{1}$ School of Physical Science and Technology \& Collaborative Innovation Center of Suzhou Nano \\ Science and Technology, Soochow University, Suzhou 215006, China. \\ ${ }^{2}$ Jiangsu Key Laboratory of Thin Films, Soochow University, Suzhou 215006, China. \\ ${ }^{3}$ College of Science, Nanjing University of Aeronautics and Astronautics, Nanjing 211106, China. \\ ${ }^{4}$ School of Engineering and Information Technology, University of New South Wales, Canberra, \\ ACT, 2600 Australia.
}

${ }^{5}$ School of Science, North University of China, Taiyuan, Shanxi, 030051, China.

\begin{abstract}
Geometrical symmetry plays a significant role in realizing robust, symmetry-protected, bound states in the continuum (BICs). However, this benefit is only theoretical in many cases since the unavoidable imperfections of fabricated samples may easily break the stringent geometrical requirements. Here we propose an essentially new approach by introducing the concept of geometrical-symmetry-free but symmetry-protected BICs, realized using the static-like environment induced by a zero-index metamaterial (ZIM). We find that robust BICs exist and are protected from the disordered distribution of multiple objects inside ZIM host by its physical symmetries rather than geometrical ones. We further show theoretically and numerically that the existence of those higher-order BICs depends only on the number of objects. By practically designing a structural ZIM waveguide, the existence of BICs is numerically confirmed, as well as their independence on the presence of geometrical symmetry. Our findings provide a new way of realizing higher-order BICs and link their properties to disorder of photonic systems.
\end{abstract}

\section{Introduction}

Recently bound states in the continuum (BICs) have attracted a growing interest in the optics community [1-6], owing to their fundamental properties and their practical applications, such as extremely strong resonances [7-9] and high quality optical lasing [10-12]. BICs are unique waves that lie in the continuum, but remain entirely confined without any radiation. BICs also feature a resonance with an infinite $Q$ factor in a spectrum, i.e., they are dark modes. Photonic BICs originating from various mechanisms [1] have been found in a number of optical systems, such as photonic crystal slabs [3, 13], dielectric gratings $[14,15]$, spheres/rod arrays [16, 17], waveguides [18, 19], and others [20]. In particular, geometrical symmetries play a significant role in the study of photonic BICs, resulting in the so-called symmetryprotected BICs that may be found in several systems $[1,13,18]$. A simple example is provided by a twodimensional (2D) waveguide structure loaded with two identical objects placed symmetrically in space, see Fig. 1a. Owing to reflection symmetry, the coupling of the discrete bound state of one symmetry class (i.e., odd symmetry) to the continuous spectrum of the other symmetry class (i.e., even symmetry) is forbidden, thus leading to the existence of symmetry-protected BICs. Physically, electromagnetic (EM) guide modes supported by the waveguide may be described by the scalar wave function $\psi_{m}(x, y)$, which obeys the Helmholtz equation: $\hat{H} \psi_{m}(x, y)=\left(\omega_{m}^{2} / c^{2}\right) \psi_{m}(x, y)$, where $\hat{H}=\nabla^{2}+\left[n^{2}(x, y)+1\right] \omega_{m}^{2} / c^{2}$ is

\footnotetext{
Correspondence and requests for materials should be addressed to Y. X. (email: ydxu@suda.edu.cn) or to L. G. (email: leigao@suda.edu.cn) or to Y.F. (email: yyfu@nuaa.edu.cn).
} 
the Hamiltonian, $n(x, y)$ is the spatially dependent refractive index profile, $\omega$ is the frequency and $c$ is the light speed. The symmetry-protected BICs are ensured by the commutation relation $\left[P_{y}, \hat{H}\right]=0$, where the parity operator $P_{y}$ is defined by the transformation $y \rightarrow-y$, and the equality $n(x, y)=n(x,-y)$ is guaranteed by the reflection symmetry.

The advantage of geometrical-symmetry-protected BICs is their robustness. However, this advantage often comes at a cost: the geometry of the system should be precisely controlled to ensure the symmetry of continuous waves and bound states in the system. A slight deviation from the required geometric structure may break the symmetry, leading to $\left[P_{y}, \hat{H}\right] \neq 0$ and, in turn, to the loss of robustness. In practice, deviations from the exact symmetry are unavoidable, due to imperfections in the fabrication technology. This is true in particular at higher operating frequencies, such as $\mathrm{THz}$ or in the visible spectrum. As a result, the advantage of robustness due to geometrical symmetry is easily lost and it is only of theoretical significance. It is thus very relevant to investigate any mechanism leading to symmetry-protected BICs which are insensitive to the geometry of the system. Although topological photonic crystals have been previously proposed to realize topological Fano resonances (i.e., quasi-BICs) robust to geometrical imperfections [21], the topological photonic crystals themselves are governed by the symmetry of geometric lattice, leading to similar control problems on the system's geometry. Therefore, how to achieve robust BICs in a system with no exact geometrical symmetry is still an open question.

Due to their static-like field distribution, zero-index metamaterials (ZIMs) [22] make it possible to realize numerous optical phenomena [23], such as squeezing wave energy [24, 25], tailoring wavefront [26-28], total transmission and reflection [29, 30] and few others [31-33]. In particular, the concept of photonic doping [34] has been proposed to tailor the effective material parameters of a 2D ZIM host doped with macroscopic dielectric objects. In fact, the effective permeability $\mu_{e}$ of the doped ZIM may be significantly modified, while maintaining its effective permittivity unchanged (i.e., $\varepsilon_{e} \cong 0$ ) for traverse magnetic (TM) waves. Regardless of the location, size and number of the doping objects, the composite structure is equivalent to a uniform system with constant index profile (i.e., $C=\sqrt{\varepsilon_{e} \mu_{e}}$ ). Therefore, although a doped ZIM may have no geometric symmetry, the condition $\left[P_{y}, \hat{H}\right]=0$ may be valid because of $\hat{H}=\nabla^{2}+(C+1) \omega_{m}^{2} / c^{2}$. In other words, the reflection symmetry is preserved by the physical ZIM homogenization at the macroscopic scale. Given $N$ identical objects immersed in a ZIM, the reflection symmetry holds even if they are places at arbitrary random locations. This fact provide the basis to design a system with BIC based on physical symmetry rather than geometric one.

In this work, we introduce the concept of geometric-symmetry-free but physical-symmetry-protected BICs using ZIM. By designing and studying a ZIM host embedded with $N \geq 2$ objects, we demonstrate the existence of robust geometric-symmetry-free BICs, resulting from a nontrivial zero value of the total effective magnetic flux in the $N$ objects. In particular, such BICs could be realized 
regardless of the external shapes and specific positions of the objects in the host. More interestingly, we find that using $N$ doping objects enables higher-order BICs, and derive an analytical formula for the $N$ dependent $Q$ factor. Specifically, the $N$ objects can produce $(N-1)$-fold degenerate BICs. These results are very different from those reported previously for ZIM-based optical systems [35, 36], where the ZIMs act as hard walls spatially separating the bound states from the extended ones. Our results pave the way to study the higher-order BICs and the associated physics using a novel approach.

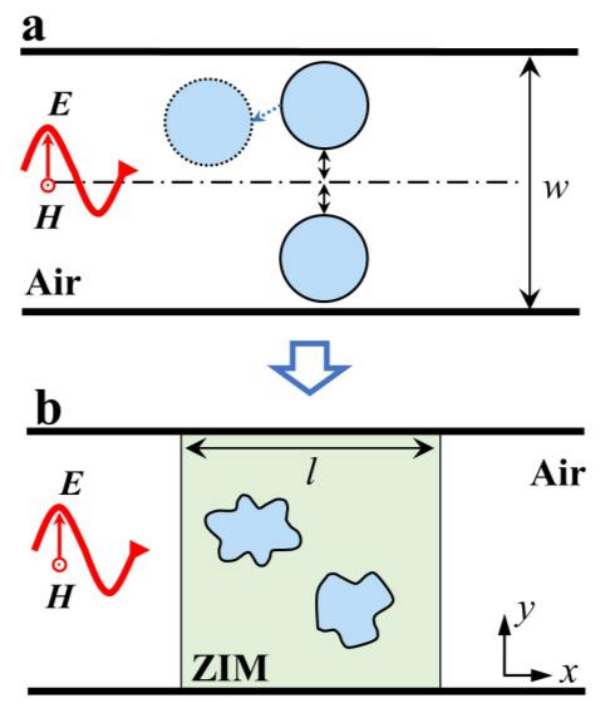

Figure 1. Geometric-symmetry-free BICs induced by near-zero index media in a waveguide. a, A twodimensional (2D) waveguide structure with reflection symmetry for symmetry-protected BICs, where two identical objects (blue circle with solid boundary) are placed symmetrically in space. If one object is shifted (the blue circle with dashed boundary) the BIC disappears. b, The proposed geometricsymmetry-free BICs in a waveguide with near-zero index medium, insensitive to the shapes and distributions of the doping objects.

\section{Results}

Models and theory. To illustrate our approach, we start our discussion from a typical 2D waveguide system (Fig. 1a). The upper and lower boundaries of the waveguide are perfect electrical conductors (PECs). For TM polarization (the magnetic field is along the $z$-direction), the waveguide supports two continuous waves (guide modes) with even and odd symmetry relative to the $x$-axis. Those odd modes have a cutoff frequency. If we introduce two identical objects with reflection symmetry, we obtain two bound modes localized near the objects, which themselves show even and odd symmetry, respectively. The odd bound mode is embedded in the continuum of even guided mode, which guarantees a robust BIC mode [1]. However, when one of the object is spatially shifted (the circle with dashed boundary in Fig. 1a), the reflection symmetry of the system is lost, leading to the disappearance of the robust BIC mode. In the following, we are going to show that such BIC mode may be preserved in a generic ZIM, even if the external shapes of these objects are arbitrary changed (see Fig. 1b).

Let us assume that $N$ objects of arbitrary shape are randomly placed in a ZIM host, and that they are nonmagnetic dielectrics with permittivity $\varepsilon_{d}$. To uncover the underlying physics of BICs in this kind of systems, impedance matched is assumed in the ZIM, i.e., $\varepsilon_{1}=\mu_{1} \rightarrow 0$. Following Ref. [30], we have that 
the transmission coefficient of the ZIM waveguide system is given by

$$
T=\frac{1}{1-\left(i \omega / 2 w H_{1}\right) \sum_{i=1}^{N} \oint_{\partial C_{i}} \vec{A}_{i} \cdot d \vec{l}},
$$

where $\vec{A}_{i}$ is the magnetic potential at the boundary $\partial C_{i}$ of the $i$-th objects, $w$ is the width of ZIM. The term $\oint_{\partial C_{i}} \vec{A}_{i} \cdot d \vec{l}$ represents the magnetic flux inside the $i$-th object. For the sake of simplicity, let us consider a cylindrical shape first. Then the magnetic flux inside the $i$-th cylinder can be expressed as

$$
\varphi_{i}=\oint_{\partial C_{i}} \vec{A}_{i} \cdot d \vec{l}=\frac{2 \pi H_{1}}{\omega} \frac{J_{1}\left(k_{2} R_{i}\right) R_{i}}{J_{0}\left(k_{2} R_{i}\right) \sqrt{\varepsilon_{d}}}
$$

Here $H_{1}$ is a local magnetic field in the ZIM and $R_{i}$ is the radius of the $i$-th cylinder. From Eq. (1) and Eq. (2), we see that the transmission coefficient depends on the properties of the object, but it is independent on the location of the objects in the ZIM.
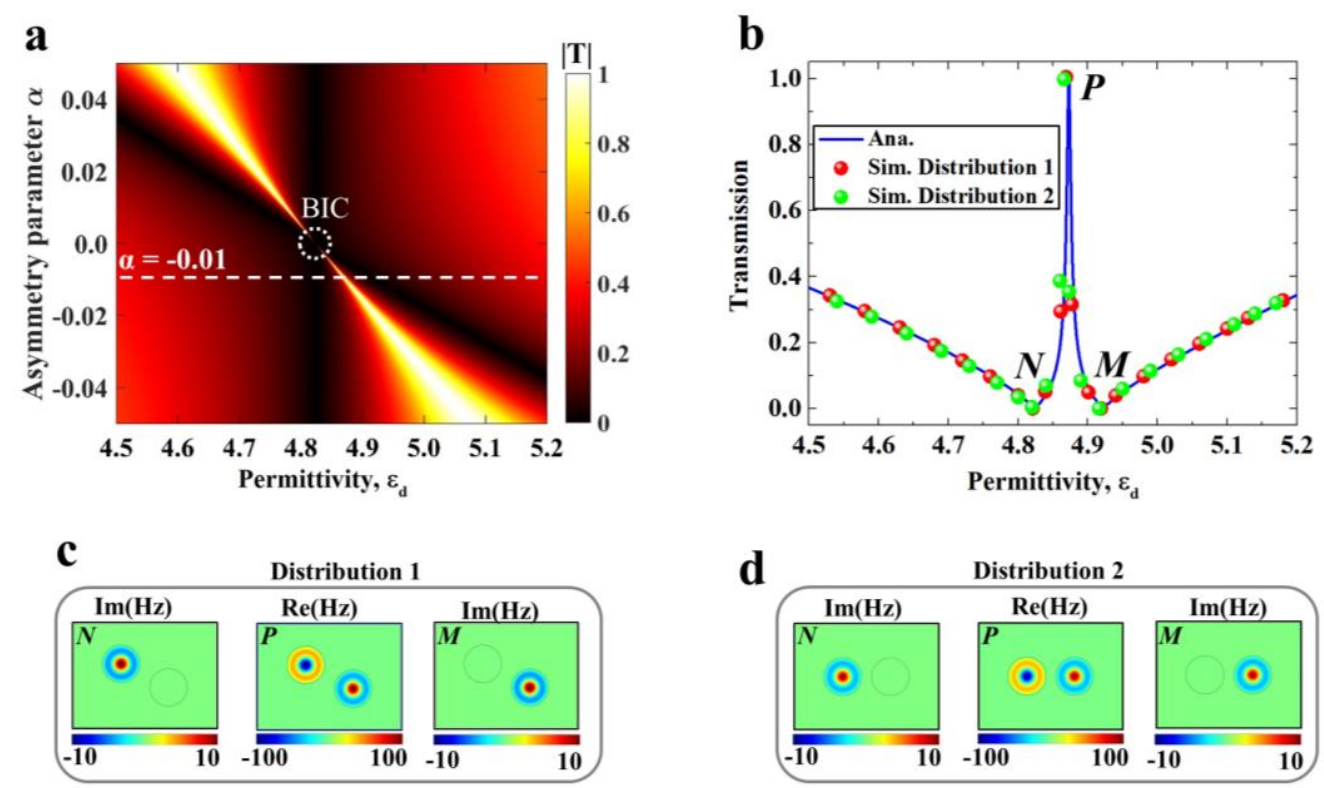

Figure 2. BIC illustrations. a, 2D map of the transmission coefficient as a function of the permittivity $\varepsilon_{d}$ and the asymmetry parameter $\alpha$. An ideal BIC occurs at $\alpha=0$ and $\varepsilon_{d}=4.82$ (the white dash circle). b, Transmission spectrum for $\alpha=-0.01$. The solid blue curve denotes the analytical results. The red and green circles represent the simulated results for the two different distributions. The (simulated) distribution of magnetic field for distribution 1 and distribution 2 are shown in panels $\mathbf{c}$ and d, respectively. $M, N$ and $P$ correspond to the two transmission dips and the peak, respectively. In the numerical simulations, the incident magnetic field is $1 \mathrm{~A} / \mathrm{m}$ and the ZIM permittivity is set to $\varepsilon_{1}=\mu_{1}=10^{-4}$. The other relevant parameters are: $R_{1}=8 \mathrm{~mm}, w=44 \mathrm{~mm}, l=60 \mathrm{~mm}$ and the working frequency is $15 \mathrm{GHz}$.

Geometry symmetry-free BICs. We first consider the simple case of two objects in the ZIM (i.e., $N=2$ ). Figure 2a shows the (analytical) results for the transmission coefficient as a function of $\varepsilon_{d}$ and $\alpha$, 
where $\alpha=\left(R_{2}-R_{1}\right) / R_{1}$ is the asymmetry parameter. In the analysis, the size of one object is fixed ( $R_{1}=8 \mathrm{~mm}$ ), and the other one is varied. As it is apparent from Fig. 2a, for a fixed $\alpha$, a transmission resonance emerges as $\varepsilon_{d}$ changes. In particular, for $\alpha \rightarrow 0$, such transmission peak becomes extremely sharp, and the corresponding quality factor diverges $Q \rightarrow \infty$. However, for $\alpha=0$ the transmission peak turns into a dip. This is a typical signature of a BIC mode, i.e. a resonance with zero linewidth. Moreover, Fig. $2 \mathrm{~b}$ shows the transmission spectrum for $\alpha=-0.01$, corresponding to a quasiBIC mode. An electromagnetically induced transparency (EIT)-like behavior appears, with a peak occurring at $\varepsilon_{d}=4.86$, and two valleys at $\varepsilon_{d}=4.82$ and $\varepsilon_{d}=4.92$, respectively. We validate our results by numerical simulations with objects at different locations, see red and green circles in Fig. 2b. The numerical results agree with the analytical ones, and the EIT-like behavior is preserved regardless of the locations of the two dielectric rods. Physically, these two valleys are stemming from monopole mode resonance occurring in either the object with $R_{1}$ (the left patterns in Fig. 2c, d) or the object with $R_{2}$ (the right patterns in Fig. 2c, d). For the transmission peak, monopole mode resonances coincide for the two objects, yet out of phase (the middle patterns in Fig. 2c, d). Note that such quasi-BIC mode leads to great field enhancement inside two objects: the EM field in the center is increased by $>100$ times compared to the incident field. Hence, the revealed (quasi)-BIC mode provides an alternative way to enhance the optical nonlinear response [37, 38].

To reveal the underlying physical mechanism, responsible for the appearance of BIC modes, we define a normalized magnetic flux for each objects, i.e., $\Phi_{i}=\varphi_{i} \omega /\left(2 \pi H_{1}\right)$, where the normalized factor $\omega /\left(2 \pi H_{1}\right)$ is the same for all the objects. Figure 3a shows $\Phi_{i}$ as a function of $\varepsilon_{d}$ for the two objects. For each object, the induced $\Phi_{i}$ changes with $\varepsilon_{d}$, featuring a strong resonance. The induced magnetic flux is pointing in opposite directions before and after this resonance, marked by the symbols "+" and "-" in the plot. Due to the different radii of two objects, their peaks do not overlap and are located at $\varepsilon_{d}=4.82$ and $\varepsilon_{d}=4.92$, respectively. These two values are consistent with the two transmission valleys in Fig. 2b. Between these two resonances, there is an intermediate region (the yellow area), in which the induced magnetic fluxes of these two objects have opposite directions. In particular, when the amplitudes of two magnetic fluxes are equal (i.e., at the crossing point), the total magnetic flux is zero, i.e., $\sum \Phi_{i}=0$ while $\Phi_{i} \neq 0$. The permittivity corresponding to this crossing point is exactly the same as that for the transmission peak in Fig. 2b. For vanishing asymmetry parameter $(\alpha \rightarrow 0)$, the two resonance peaks tend to overlap and the middle region shrinks, yet the total magnetic flux is always zero, thus ensuring the occurrence of the transmission peak. One may conjecture that for $\alpha=0$ the two resonance peaks are going to coincide exactly at $\varepsilon_{d}=4.82$, and that there are two-fold degenerate states in the two objects, i.e., one state with infinite total flux and the other one with zero total flux. In particular, the state with zero total flux is a dark mode, leading to ideal BIC. Thus, the BIC mode discussed above is physically related to the nontrivial zeros of the total magnetic flux.

In order to validate this picture, we analyze the eigenmodes of the doped ZIM numerically and analytically (see Supplementary Note 1). For $\alpha=0$, there are two eigenmodes at the eigenfrequency 15 $\mathrm{GHz}$, and Fig. $3 \mathrm{~b}$ shows the corresponding field distributions, where the color contour is the out-of-plane magnetic field, and the magenta cones denote the directions of the in-plane electric field. Note that due to the absence of any geometric symmetry, we cannot define exactly the symmetry of the two eigenmodes. However, if we focus on the magnetic field distributions in the two objects, we can see that one of these 
two eigenmodes exhibits symmetry-like features, e.g. in-phase magnetic field profiles in the two objects, and vortex-like electric fields in the two objects having the same rotation directions. The other one is the anti-symmetric-like mode, as suggested by the out-phase magnetic field profiles in two objects, and by the opposite rotation directions of the electric fields. The homogenous magnetic field inside the ZIM background highly depends on the symmetry of eigenmode. For the symmetric case, the magnetic field inside the ZIM is non-zero. As a result, the symmetric-like mode can efficiently couple with the incident filed from outside, i.e. it is a bright (leaky) mode, leading to the transmission valleys in the spectrum. For the anti-symmetric one, the magnetic field inside the ZIM background is exactly zero, and the mode completely decouples from the incident field, i.e. it can be regarded as a dark mode or bound mode, with unit transmission. This dark mode is actually the BIC mode. Due to the quasi-static feature and constant field in the ZIM, these eigenmodes can usually exist independently of their specific distribution. Thereby they can be distributed arbitrarily. Note that the above results can be extended to a ZIM medium doped with dielectric objects of arbitrary shape. We numerically studied several cases of dielectric objects with square, triangle, and arbitrary external shapes to confirm this feature. The corresponding results are displayed respectively in Supplementary Figs. 3-6. In all cases, similar BICs may be found, always coming from the zero total flux in the two objects with the anti-symmetry-like mode state.

a

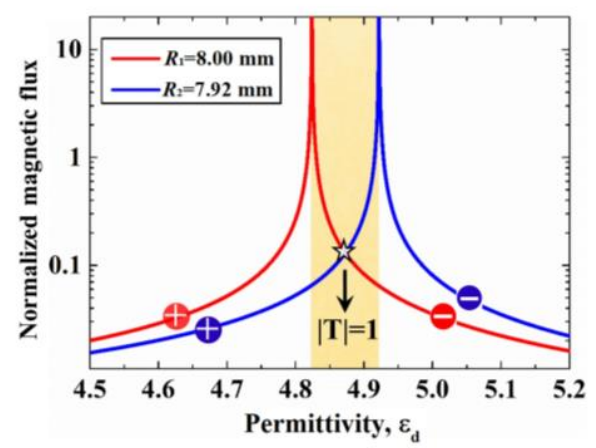

b

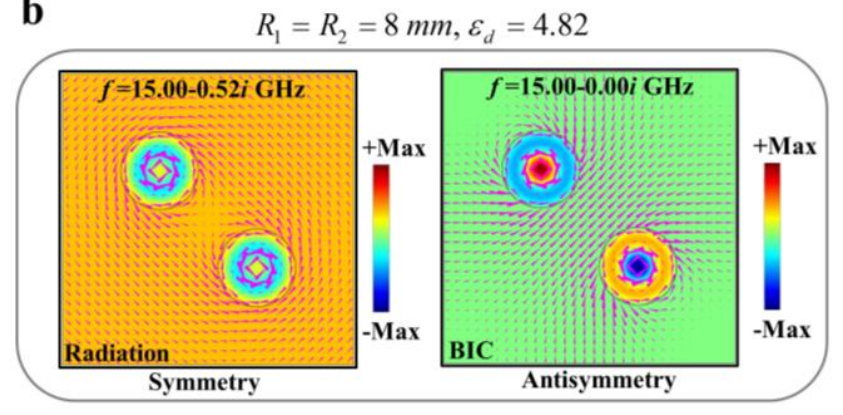

Figure 3. Normalized magnetic flux and eigenmode analysis. a, Normalized magnetic flux in each object as a function of $\varepsilon_{d}$, "+" and "-" denote the direction of magnetic flux. b, Eigenmode analysis for a ZIM environment with two identical objects with $R_{1}=R_{2}=8 \mathrm{~mm}$ and $\varepsilon_{d}=4.82$. Filling contours are outof-plane magnetic field, and magenta cones represent the in-plane electric field.

Higher-order BICs. Following the results obtained with two objects, we turn attention to the general case, i.e., a ZIM with an arbitrary number of objects ( $N$ groups of objects). Interestingly, we find that the presence of $N$ objects leads to $(N-1)$-th order BIC. For simplicity, let us consider the case $N=3$ to illustrate the phenomenon. The analytical and numerical results for transmission spectrum are shown in Supplementary Fig. 7, where the radius of the first object is fixed $R_{1}=8 \mathrm{~mm}$, and the other ones have slightly different radii, i.e., $R_{2}=7.92 \mathrm{~mm}$ and $R_{3}=8.08 \mathrm{~mm}$. As it is apparent from the plot, two EITs may be seen in the transmission spectrum, stemming from the zero total magnetic flux in the three objects, i.e., $\sum \Phi_{i}=0$. Figure 4 a shows $\Phi_{i}$ as a function of $\varepsilon_{d}$ for the three objects. There are three resonant peaks, which divide the whole range into four areas. There are two intermediate regions (colored regions in the plot), and there is a point where $\sum \Phi_{i}=0$ in each region. This means there are two quasi-BIC modes in the $N=3$ case. As $\alpha_{2} \rightarrow 0$ and $\alpha_{3} \rightarrow 0$, these two quasi-BIC modes coincide, leading to a 
high-order BIC mode. Further, the eigenmodes of the ZIM background with three identical objects (corresponding to $\alpha_{i}=0$ ) have been analyzed, and their corresponding field distributions are shown in Fig. 4b: three eigenmodes appear at the eigenfrequency $15 \mathrm{GHz}$. Differently from the $N=2$ case, the symmetry of eigenmodes in this situation cannot be exactly defined, because the magnetic field distributions in three objects are irregular. Instead, these three modes can be distinguished by the intensity of the magnetic field in ZIM, i.e., one bright mode with non-zero magnetic field in the ZIM, and two dark modes with zero magnetic field in the ZIM. In particular, both dark modes fully decouple from the external excitation and enable two-fold degenerate BIC. Strictly speaking, the symmetry of BICs in a general configuration cannot be defined, except for the case of $N=2$. Accordingly, for $N$ objects in ZIM, there are $N-1$ groups of EITs and $N-1$ groups of quasi-BIC modes (linked to the condition $\sum \Phi_{i}=0$ ). Consequently, $N$ identical objects in the ZIM induce $(N-1)$-fold degenerate BICs.
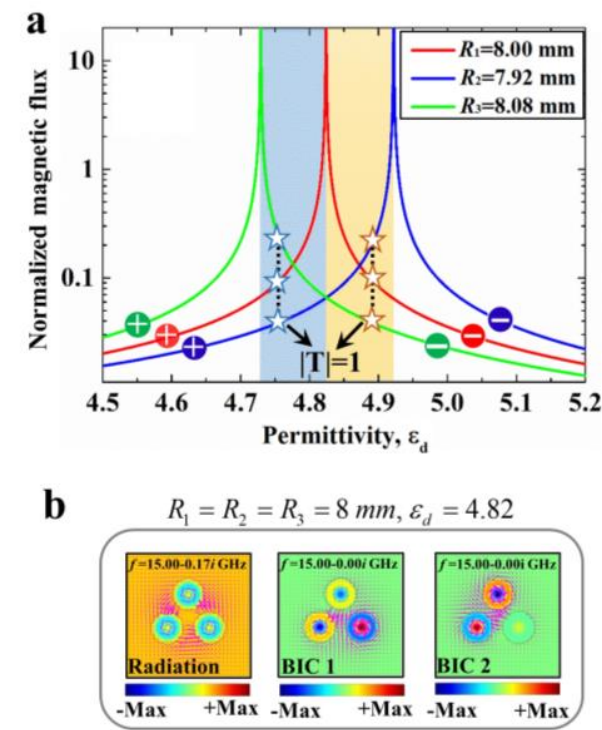

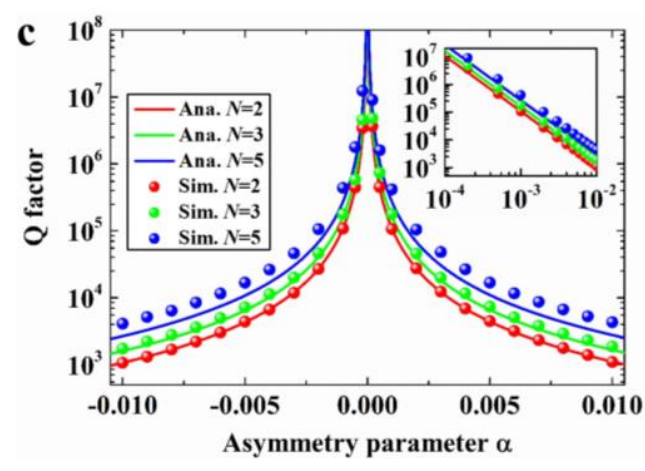

d

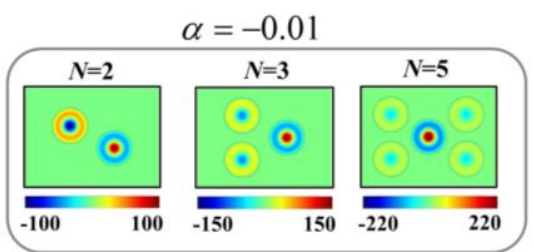

Figure 4. Higher-order optical BICs. a, Normalized magnetic flux as a function of $\varepsilon_{d}$ for a ZIM with three objects having $R_{1}=8 \mathrm{~mm}, R_{2}=0.99 R_{1}$ and $R_{3}=1.01 R_{1} . \mathrm{b}$, The eigenmodes for a ZIM host with three identical objects ( $R_{1}=R_{2}=R_{3}=8 \mathrm{~mm}$ and $\varepsilon_{d}=4.82$ ) at $15.0 \mathrm{GHz}$. c, $Q$ factor as a function of the asymmetry parameter for different values of $\mathrm{N}$ (the number of objects). The solid curves and circles denote the analytical and numerical results, respectively. The inset shows the same results in log scale. d, Out-of-plane magnetic field at total transmission for $\alpha=-0.01$. Higher-order quasi-BICs are observed.

The presence of higher-order BIC is further confirmed by looking at the $Q$ factor for the case of $N$ objects and $\varepsilon_{d}=4.82$. For the sake of simplicity, we assume that the $N$ objects consist of $(N-1)$ identical objects with fixed radius $R_{a}=8 \mathrm{~mm}$ and one object with a variable radius $R_{b}$. After some calculations (see Supplementary Note 2), the $Q$ factor of the quasi-BIC is derived as,

$$
Q=\frac{2 \pi k_{0} R_{b}^{2}}{\sqrt{3} w s_{v}^{2}} \frac{N}{\alpha^{2}},
$$

where $s_{v}$ is the $v$-th solution of $J_{0}(x)=0$ and $\alpha=\left(R_{b}-R_{a}\right) / R_{a}$ is the asymmetry parameter. For the 
current case ( $\varepsilon_{d}=4.82, R_{a}=8 \mathrm{~mm}$ and $f=15.0 \mathrm{GHz}$ ), the second solution $(v=2)$ corresponds to the monopole resonance in the object, marked as $\mathrm{TM}_{02}$ mode. Clearly, $Q$ factor is proportional to $N$ and inversely proportional to $\alpha^{2}$. Figure 4c shows the relation between $Q$ and $\alpha$ for different $N$ (red, green, and blue curves refer to the cases of $N=2,3$ and 5 , respectively). The analytical results are calculated using Eq. (3), and the numerical ones are obtained from COMSOL. Both analytical and numerical results agree with each other, with the slight deviation seen for large $N$ caused by the approximations used in the theoretical analysis. In particular, the condition $\alpha=0$ leads to an infinite $Q$, which proves the existence of the BICs theoretically. For $\alpha$ deviating from zero, the quasi-BICs may occur with a finite and high $Q$ factor. For a fixed $\alpha \neq 0$, the $Q$ factor may be largely increased by adding objects with $R_{a}$, leading to further enhancement of EM fields confined inside the objects (see Fig. 4(d)). This result is further confirmed by eigenmode analysis in the frequency domain (see Supplementary Fig. 2). This finding provides a feasible way to enhance the dielectric sensitivity of optical structures to external perturbations by simply incorporating multiple objects with the same size.

a
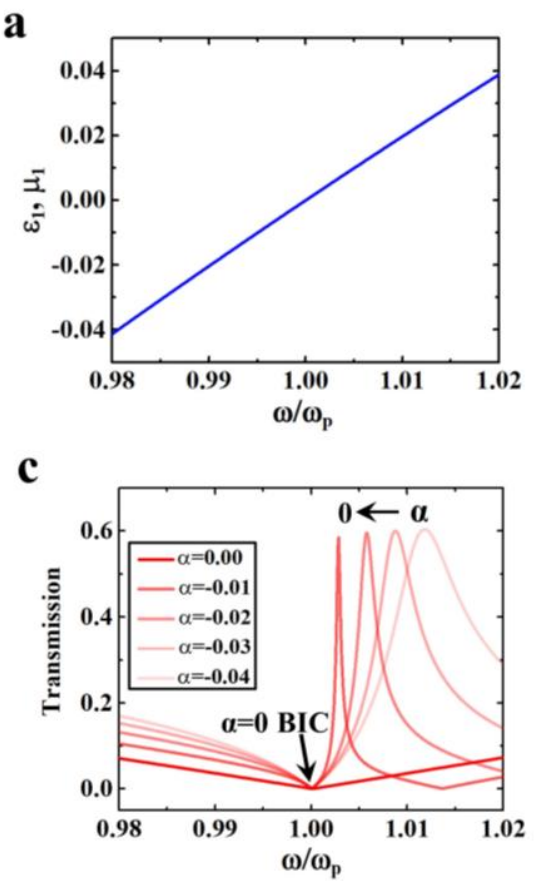
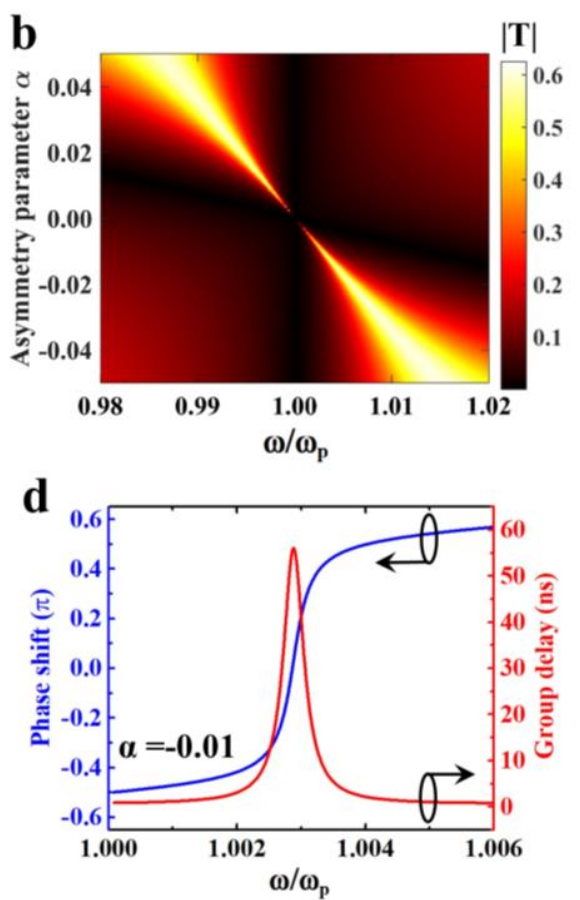

Figure 5. BIC frequency response in a dispersive ZIM with $\mathbf{N = 2}$ objects. a, Drude model for ZIM media. b) Transmission coefficient as a function of frequency and $\alpha$ for fixed $R_{1}=8 \mathrm{~mm}$. c, Transmission coefficient as a function of frequency: quasi-BIC turns to BIC as $\alpha \rightarrow 0$. d, Phase shift in the transmitted light and group delay due to quasi-BIC.

The frequency response of BICs. It is known that the frequency response of a ZIM is usually narrowband, i.e. ZIM-based devices only work at a single frequency. In spite of this, the BIC discussed above can still be found in the spectrum because the BIC with an extreme Q factor has a narrower bandwidth response compated to that of the ZIMs. To illustrate this point, we assume the ZIM to be a Drude-like material with parameters $\varepsilon_{1}=\mu_{1}=1-\omega_{p}^{2} /[\omega(\omega+i \gamma)]$, where $\omega_{p}$ is plasma frequency and $\gamma$ is the damping. To make the discussion simpler, we set $\omega_{p}=2 \pi \times 15 \times 10^{9} \mathrm{rad} / \mathrm{s}$ and ignore the 
damping, i.e., set $\gamma=0$. Figure 5a shows the corresponding dispersion relationship, in which for $0.98 \leq \omega / \omega_{p} \leq 1.02$, the material features a near-zero profile, ranging from -0.02 to 0.02 . With this dispersion, Figure $5 \mathrm{~b}$ shows the calculated transmission coefficient as a function of $\omega$ and $\alpha$ for $N=2$ case. We can clearly observe BICs features similar to that in Fig. 2a. For a fixed $\alpha$, a transmission resonance of quasi-BIC emerges in the spectrum close to $\omega / \omega_{p}=1$, and as $\alpha \rightarrow 0$, it becomes shaper and eventually disappears (see Fig. 5c). These results further confirm that the case $\alpha=0$ is associated with an ideal BIC. At the same time, this form of BIC actually provides a new mechanism to slow the speed of light, thus enhancing the interaction between light and matter. Figure $5 \mathrm{~d}$ shows the calculated transmission phase shift $\phi(\omega)$ (blue curve) and the delay time (the red curve) by using the relation $\tau=\partial \phi(\omega) / \partial \omega$ [39] for a quasi-BIC mode with $\alpha=-0.01$. A noticeable delay is seen at $\omega / \omega_{p} \approx 1$. As $\alpha \rightarrow 0$, the phase shift get steeper and steeper, and when $\alpha=0$ corresponding to an ideal BIC, the phase shift abruptly change at $\omega / \omega_{p} \approx 1$, just like a step function, which means an infinite delay time, at least theoretically.

Practical design and demonstration of ZIM-based BICs using a structural waveguide. Realistic ZIMs are lossy, and this fact may erase almost all ZIM-based effects. However, the loss of dielectrics or metals can be negligible at microwave frequencies, and there also are some methods to realize a lossless ZIM, such as the Dirac-cone like photonic crystals [40]. Alternatively, effective ZIMs with matched impedance may be realized in waveguide systems [34] consisting of two rectangular waveguides (port 1 and port 2) and a waveguide junction. By operating at a working frequency close to the cutoff frequency of each guided mode (e.g., $\mathrm{TE}_{10}$ mode), the effective permittivity of the waveguide junction is that of an epsilon-near-zero (ENZ) medium, and its effective permeability could be tuned by doping the waveguide junction with suitable dielectric rods, thus realizing and effective ZIM with matched impedance in (see Fig. 6a). In our design, the height of the waveguide, i.e., the separation of two parallel metallic plates in the $z$-axis, is $H=\lambda_{0} / 2$, and the operating frequency is $f_{0}=2.5 \mathrm{GHz}$, corresponding to the cutoff frequency of $\mathrm{TE}_{10}$ mode. The cross-section of the waveguide junction is a square with side $L=240 \mathrm{~mm}$, and the width of the input (output) waveguide port is $W=42 \mathrm{~mm}$. A dielectric material (Teflon, $\varepsilon_{r}=2$, blue areas in Fig. 6a) is used to fill in the input and output waveguides, such that $\mathrm{TE}_{10}$ mode above the cutoff frequency is supported. A silicon rod is inserted in the waveguide junction to tune the effective permeability, and 16 metallic wires with a diameter of $3.18 \mathrm{~mm}$ are placed (equally spaced) around it to form a circle with of radius $26.8 \mathrm{~mm}$, which allows us to avoid the excitation of other guided modes. Since the setup is aimed at demonstrating quasi-BIC in this ZIM environment, at least additional two objects (e.g., two rods) are required. To this aim, two identical circles (each one made of 16 metallic wires) are also involved in the waveguide junction (see Fig. 6a). 


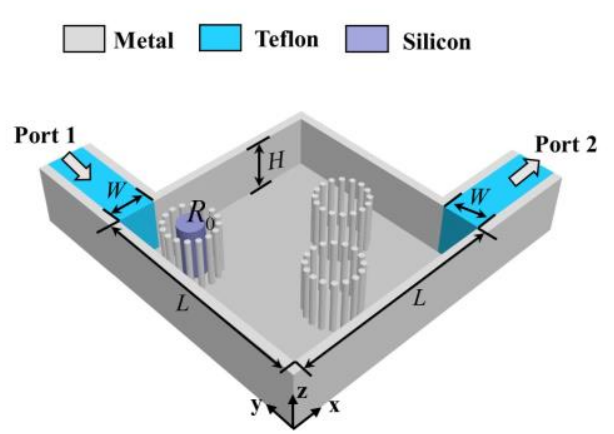

c

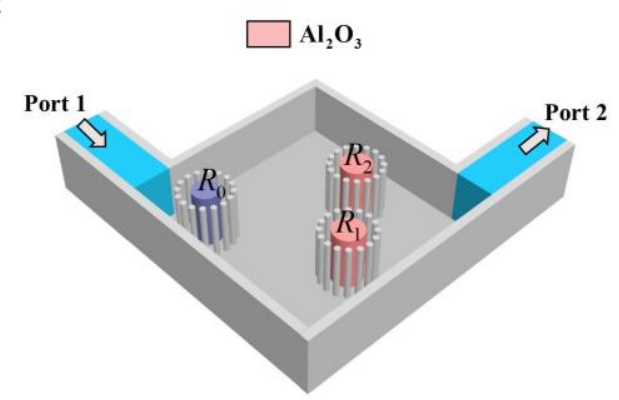

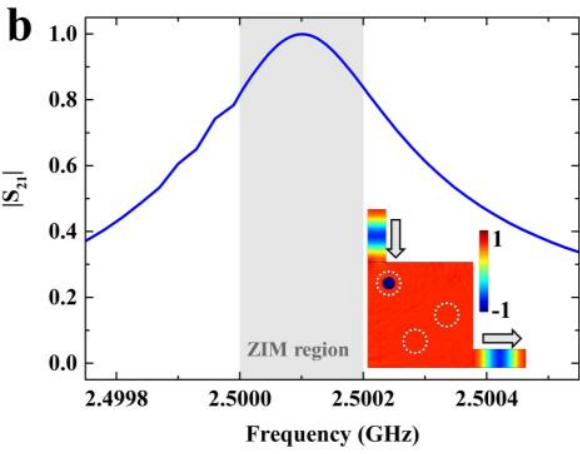

d

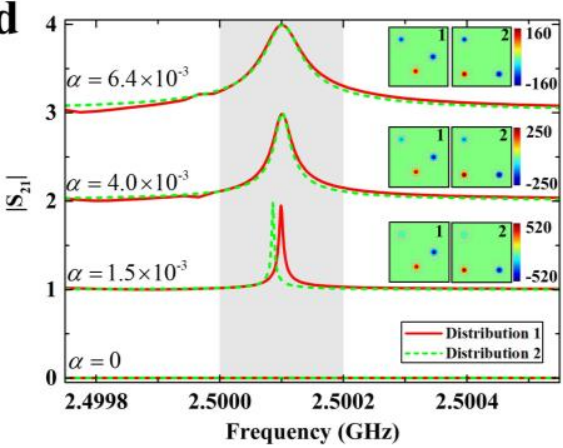

Figure 6. Experimental design for ZIM-induced BIC in a metallic rectangular waveguide system. a, Schematic diagram of a metallic rectangular waveguide system with ZIM. The upper parallel metallic plate is hidden to show more clearly the internal structure. b, Numerically calculated transmission spectrum $\left|S_{21}\right|$ of the designed waveguide system with ZIM. The grey region shows the parameter region of $\varepsilon_{\text {eff }} \approx \mu_{\text {eff }} \approx 0$. The inset shows the magnetic field in the middle plane $(z=H / 2)$ at the resonant transmission. $\mathbf{c}$, Schematic diagram of the metallic rectangular waveguide system for observing BIC, where two dielectric rods (aluminium oxide) are added in the designed waveguide system with ZIM. d, Transmission spectrum $\left|S_{21}\right|$ for the aluminium oxide rods with different $\alpha$. The red solid and green dashed curves denote the two different distributions of aluminium oxide rods. The insets show the corresponding magnetic field patterns in the waveguide junction (square area) with doped rods. Simulation parameters of $R_{1}$ and $R_{2}: \quad \alpha=6.4 \times 10^{-3} \quad\left(R_{1}=16.176 \mathrm{~mm}\right.$ and $\left.R_{2}=16.280 \mathrm{~mm}\right)$, $\alpha=4.0 \times 10^{-3} \quad\left(R_{1}=16.195 \mathrm{~mm}\right.$ and $\left.R_{2}=16.260 \mathrm{~mm}\right), \quad \alpha=1.5 \times 10^{-3} \quad\left(R_{1}=16.215 \mathrm{~mm}\right.$ and $R_{2}=16.240$ $\mathrm{mm}), \quad \alpha=0 \quad\left(R_{1}=R_{2}=16.227 \mathrm{~mm}\right)$. In all simulations, the metals are perfect electric conductor (PEC);

the material parameters are $\varepsilon_{r}=2, \varepsilon_{S i}=11.7, \varepsilon_{d}=9$.

Based on such a waveguide configuration, we numerically obtain the transmission spectrum $\left(\left|S_{21}\right|\right)$, which is in Fig. $6 \mathrm{~b}$, where the radius of silicon rod is set to $R_{0}=14.087 \mathrm{~mm}$. High transmission, beyond $80 \%$, is found in a narrow band (grey region) from 2.5 to $2.5002 \mathrm{GHz}$, caused by the near-zero index region $\left(\varepsilon_{\text {eff }} \approx \mu_{\text {eff }} \approx 0\right)$ (see the analytical results in Supplementary Note 3 and Supplementary Fig. 8). In particular, a unity transmission occurs at $2.5001 \mathrm{GHz}$, which means that an effective ZIM with matched impedance $\left(\varepsilon_{\text {eff }}=\mu_{e f f} \rightarrow 0\right)$ is obtained as indicated by Supplementary Fig. 8. In addition, the inset in Fig. $6 \mathrm{~b}$ shows the corresponding magnetic field pattern in the waveguide at $2.5001 \mathrm{GHz}$. Total 
transmission is observed, and the constant phase distribution appears in the waveguide junction, also implying that an effective ZIM with matched impedance is achieved. Then, we add two dielectric rods (aluminium oxide) with $\varepsilon_{d}=9$ into the waveguide, as shown in Fig. 6c (distribution 1). By changing the asymmetry $\alpha$ of the two aluminium oxide rods, the transmission spectra shown in Fig. $6 \mathrm{~d}$ are obtained (red curves). For $\alpha \rightarrow 0$, the transmission peak occurring at $2.5001 \mathrm{GHz}$ becomes narrower, which means that a quasi-BIC is obtained with a higher $\mathrm{Q}$ factor. The corresponding magnetic field distributions in the waveguide junction are shown in the insets of Fig. 6d, where the corresponding field distribution in two aluminium oxide rods are out of phase, and their amplitudes are stronger for $\alpha \rightarrow 0$. Next, we change the locations of $R_{1}$ and $R_{2}$ (distribution 2). As can be seen from the green dashed curves, the ultrasharp spectra are almost the same as their counterparts coming from distribution 1 . This confirms that the ZIM-based BIC are not affected by structural disorder. These results are consistent with the ideal ones reported in Fig. 2. For $\alpha=0$, the resonant peak disappears in the transmission spectrum, indicating the presence of an ideal BIC.

\section{Conclusions}

In conclusion, upon exploiting the static-like environment of ZIM in 2D waveguide, we have theoretically proposed and numerically demonstrated the existence of symmetry-protected but geometry independent BICs. Those BIC modes are determined by the zero total magnetic flux in the objects in the ZIM host, and exist independently on the shape and position of the objects, which is a unique feature compared with previous results. Remarkably, higher-order BICs may also easily achieved by doping the ZIM host with more objects. More precisely, $N$ identical objects have been shown to induce $(N-1)$-th order BICs, and this result provides a feasible and straightforward way to manipulate $Q$ factor of quasiBIC mode. Although the existence of geometric-symmetry-free BICs has been revealed using ZIMs with matched impedance, similar results may be also obtained in an ENZ-based host (see Supplementary Note 4 and Supplementary Fig. 9). Our findings offer a new way to realize an optical BIC mode without stringent requirements on geometry of the system and enable flexible manipulation of robust, sharp resonances. We foresee promising applications in ultra-fast optical switches, filters, and sensors.

\section{Methods}

Numerical simulations. The data in Fig. 2b, Fig. 4c, and Figs. 5b-d, and the field patterns in Figs. 2c-d, Fig. 3b, Figs. 4b, d, and the insets of Figs. 6b, d were obtained using the finite element solver COMSOL Multiphysics. For eigenmode analysis, in simulations, we studied a case of a ZIM background containing two same objects of $\varepsilon_{d}=4.82$ instead. A large ZIM area replaces the infinite ZIM with $r=10 R_{1}$. We obtained two eigenfrequencies and corresponding filed pattern, as shown in Fig. 3c. Similar procedures were applied to the case of three cylinders with $\varepsilon_{d}=4.82$ and $R_{1}=R_{2}=R_{3}=8 \mathrm{~mm}$ embedded in the ZIM environment. Based on numerical calculations, we can easily get the eigenfrequency of each eigenmode and its corresponding field pattern, as shown in Fig. $4 \mathrm{~b}$.

\section{Data availability}

The data that support the findings of this study are available from the corresponding author upon reasonable request.

\section{Code availability}

The code used for the analyses will be made available upon e-mail request to the corresponding author. 


\section{Reference}

1. Hsu, C. W., Zhen, B., Stone, A. D., Joannopoulos, J. D. \& Soljačić, M. Bound states in the continuum. Nat. Rev. Mater. 1, 16048 (2016).

2. Marinica, D. C. \& Borisov, A. G. Bound states in the continuum in photonics. Phys. Rev. Lett. 100, 183902 (2008).

3. Hsu, C. W. et al. Observation of trapped light within the radiation continuum. Nature 499, 188 (2013)

4. Gomis-Bresco1, J., Artigas, D. \& Torner, L. Anisotropy-induced photonic bound states in the continuum. Nat. Photon. 11, 232-236 (2017).

5. Azzam, S. I., Shalaev, V. M., Boltasseva, A. \& Kildishev, A. V. Formation of bound states in the continuum in hybrid plasmonic-photonic systems. Phys. Rev. Lett. 121, 253901 (2018).

6. Yin, X., Jin, J., Soljačić, M., Peng, C. \& Zhen, B. Observation of topologically enabled unidirectional guided resonances. Nature 580, 467-471 (2020).

7. Krasikov, S. D., Bogdanov, A. A. \& Iorsh, I. V. Nonlinear bound states in the continuum of a onedimensional photonic crystal slab. Phys. Rev. B 97, 224309 (2018).

8. Cao, Y. et al. Giant Goos-Hänchen shift induced by bounded states in optical PT-symmetric bilayer structures. Opt. Express 27, 7857-7867 (2019).

9. Liu, Z. et al. High-Q quasibound states in the continuum for nonlinear metasurfaces. Phys. Rev. Lett. 123, 253901 (2019).

10. Hirose, K. et al Watt-class high-power, high-beam-quality photonic-crystal lasers. Nat. Photon. 8, 406-411 (2014)

11. Kodigala, A. et al. Lasing action from photonic bound states in continuum. Nature 541, 196-199 (2017).

12. Huang, C. et al. Ultrafast control of vortex microlasers. Science 367, 1018-1021 (2020).

13. Lee, J. et al. Observation and differentiation of unique high-q optical resonances near zero wave vector in macroscopic photonic crystal slabs. Phys. Rev. Lett. 109, 067401 (2012).

14. Taghizadeh, A. \& Chung, I. S. Quasi bound states in the continuum with few unit cells of photonic crystal slab. Appl. Phys. Lett. 111, 031114 (2017).

15. Bulgakov, E. N. \& Maksimov, D. N. Avoided crossings and bound states in the continuum in lowcontrast dielectric gratings. Phys. Rev. A 98, 053840 (2018).

16. Bulgakov, E. N. \& Maksimov, D. N. Bound states in the continuum and polarization singularities in periodic arrays of dielectric rods. Phys. Rev. A 96, 063833 (2017).

17. Bulgakov, E. N. \& Sadreev, A. F. High-Q resonant modes in a finite array of dielectric particles. Phys. Rev. A 99, 033851 (2019).

18. Plotnik, Y., Peleg, O., Dreisow, F., Heinrich, M. \& Nolte, S. Experimental observation of optical bound states in the continuum. Phys. Rev. Lett. 107, 183901 (2011).

19. Xu, H. \& Shi, Y. Silicon-waveguide-integrated high-quality metagrating supporting bound state in the continuum. Laser Photon. Rev. 14, 1900430 (2020).

20. Hsu, C. W. et al. Bloch surface eigenstates within the radiation continuum. Light Sci. Appl. 2, e84 (2013).

21. Zangeneh-Nejad, F. \& Romain, F. Topological Fano resonances. Phys. Rev. Lett. 122, 014301 (2019)

22. Liberal, I. \& Engheta, N. Near-zero refractive index photonics. Nat. Photon. 11, 149-158 (2017).

23. Niu, X., Hu, X., Chu, S. \& Gong, Q. Epsilon-near-zero photonics: a new platform for integrated 
devices. Adv. Opt. Mater. 6, 1701292 (2018).

24. Silveirinha, M. \& Engheta, N. Tunneling of electromagnetic energy through subwavelength channels and bends using $\varepsilon$-near-zero materials. Phys. Rev. Lett. 97, 157403 (2006).

25. Liu, R. et al. Experimental demonstration of electromagnetic tunneling through an epsilon-nearzero metamaterial at microwave frequencies. Phys. Rev. Lett. 100, 023903 (2008).

26. Enoch, S., Tayeb, G., Sabouroux, P., Guérin, N. \& Vincent, P. A metamaterial for directive emission. Phys. Rev. Lett. 89, 213902 (2002).

27. Alù, A., Silveirinha, M. G., Salandrino, A. \& Engheta, N. Epsilon-near-zero metamaterials and electromagnetic sources: Tailoring the radiation phase pattern. Phys. Rev. B 75, 155410 (2007).

28. Luo, J. et al. Arbitrary control of electromagnetic flux in inhomogeneous anisotropic media with near-zero index. Phys. Rev. Lett. 112, 073903 (2014).

29. Nguyen, V. C., Chen, L. \& Halterman, K. Total transmission and total reflection by zero index metamaterials with defects. Phys. Rev. Lett. 105, 233908 (2010).

30. Xu, Y. \& Chen, H. Total reflection and transmission by epsilon-near-zero metamaterials with defects. Appl. Phys. Lett. 98, 113501 (2011).

31. Xu, Y., Chan, C. T. \& Chen, H. Goos- Hänchen effect in epsilon-near-zero metamaterials. Sci. Rep. 5, 8681 (2015).

32. Fu, Y., Xu, Y. \& Chen, H. Zero index metamaterials with PT symmetry in a waveguide system. Opt. Express 24, 1648-1657 (2016).

33. Fu, Y., Zhang, X., Xu, Y. \& Chen, H. Design of zero index metamaterials with PT symmetry using epsilon-near-zero media with defects. J. Appl. Phys. 121, 094503 (2017).

34. Liberal, I., Mahmoud, A. M., Li, Y., Edwards, B. \& Engheta, N. Photonic doping of epsilon-nearzero media. Science 355, 1058-1062 (2017).

35. Silveirinha, M. G. Trapping light in open plasmonic nanostructures. Phys. Rev. A 89, 023813 (2014).

36. Monticone, F. \& Alù, A. Embedded photonic eigenvalues in $3 \mathrm{~d}$ nanostructures. Phys. Rev. Lett. 112, 213903 (2014).

37. Liu, Y. et al. Enhanced third-harmonic generation induced by nonlinear field resonances in plasmonic-graphene metasurfaces. Opt. Express 28, 13234-13242 (2020).

38. $\mathrm{Xu}, \mathrm{L}$. et al. Dynamic nonlinear image tuning through magnetic dipole quasi - BIC ultrathin resonators. Adv. Sci. 6, 1802119 (2019).

39. Hu, C., Schulz, S. A., Liles, A. A. \& O'Faolain, L. Tunable optical buffer through an analogue to electromagnetically induced transparency in coupled photonic crystal cavities. ACS Photon. 5, 1827 (2018).

40. Huang, X., Lai, Y., Hang, Z. H., Zheng, H. \& Chan, C. T. Dirac cones induced by accidental degeneracy in photonic crystals and zero-refractive-index materials. Nat. Mater. 10, 582-586 (2011).

\section{Acknowledgments}

This work was supported by The National Natural Science Foundation of China (grant Nos. 11974010, 11904169, 11774252, 92050104, 61705200 and 11604229); the Natural Science Foundation of Jiangsu Province (grant Nos. BK20171206 and BK20190383); the project funded by the China Postdoctoral Science Foundation (grant Nos. 2018T110540, 2020M681576); and the Priority Academic Program Development (PAPD) of Jiangsu Higher Education Institutions. 


\section{Author contributions}

Y. X. and L. G. conceived the idea. Q. Z., Y. Y, L. H. and Y. X. performed the theoretical calculation and numerical simulations. L. G., Q. W. and A. M. helped with the theoretical interpretation. Y. X., Y. F., and L. G. supervised the project. All authors discussed the results and prepared the manuscript.

\section{Additional Information}

Supplementary Information accompanies this paper at http xxxxx.

Competing interests: The authors declare no competing financial interests. 
Figures

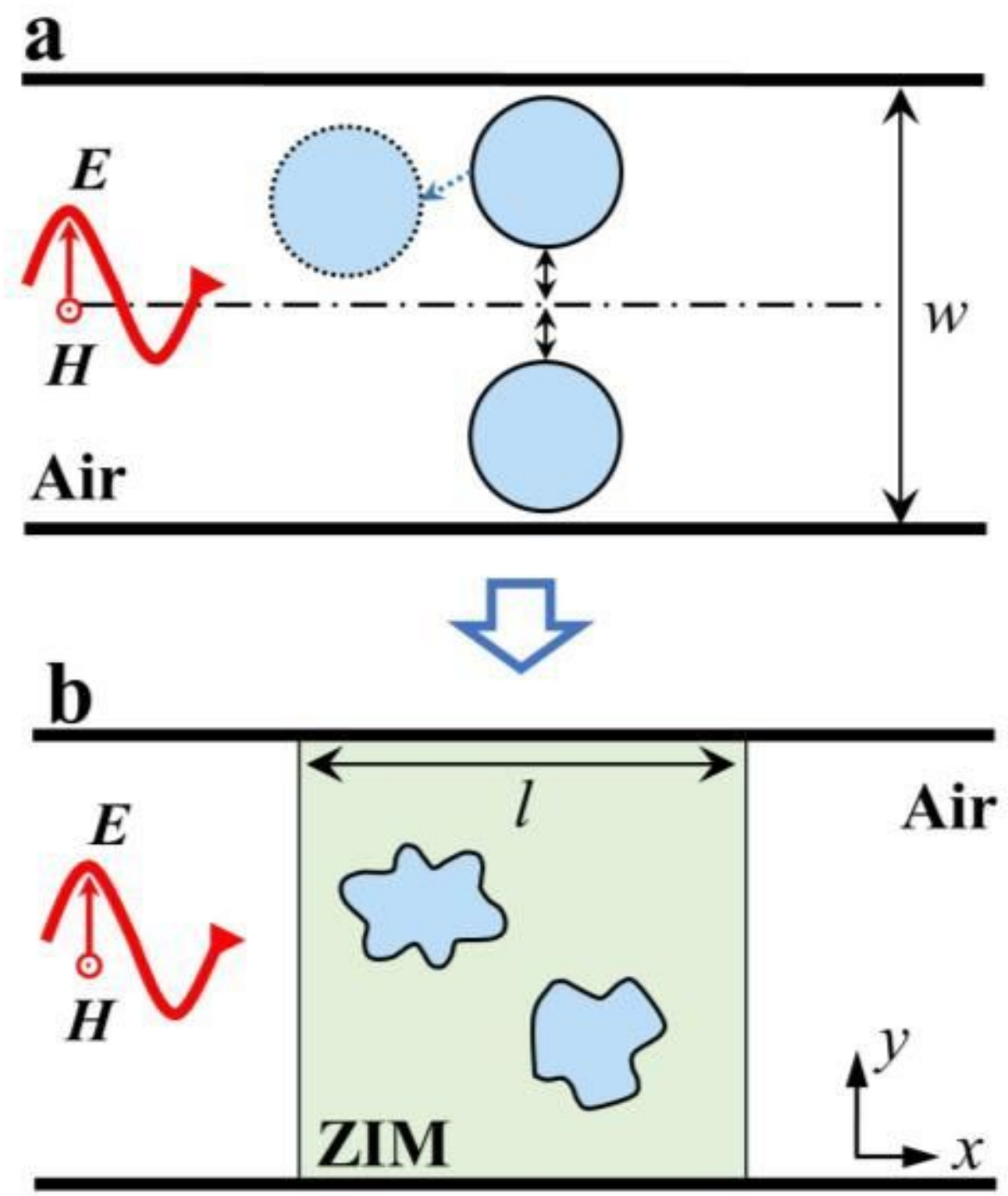

\section{Figure 1}

Geometric-symmetry-free BICs induced by near-zero index media in a waveguide. a, A twodimensional (2D) waveguide structure with reflection symmetry for symmetry-protected BICs, where two identical objects (blue circle with solid boundary) are placed symmetrically in space. If one object is shifted (the blue circle with dashed boundary) the BIC disappears. b, The proposed geometricsymmetry-free BICs in a waveguide with near-zero index medium, insensitive to the shapes and distributions of the doping objects. 

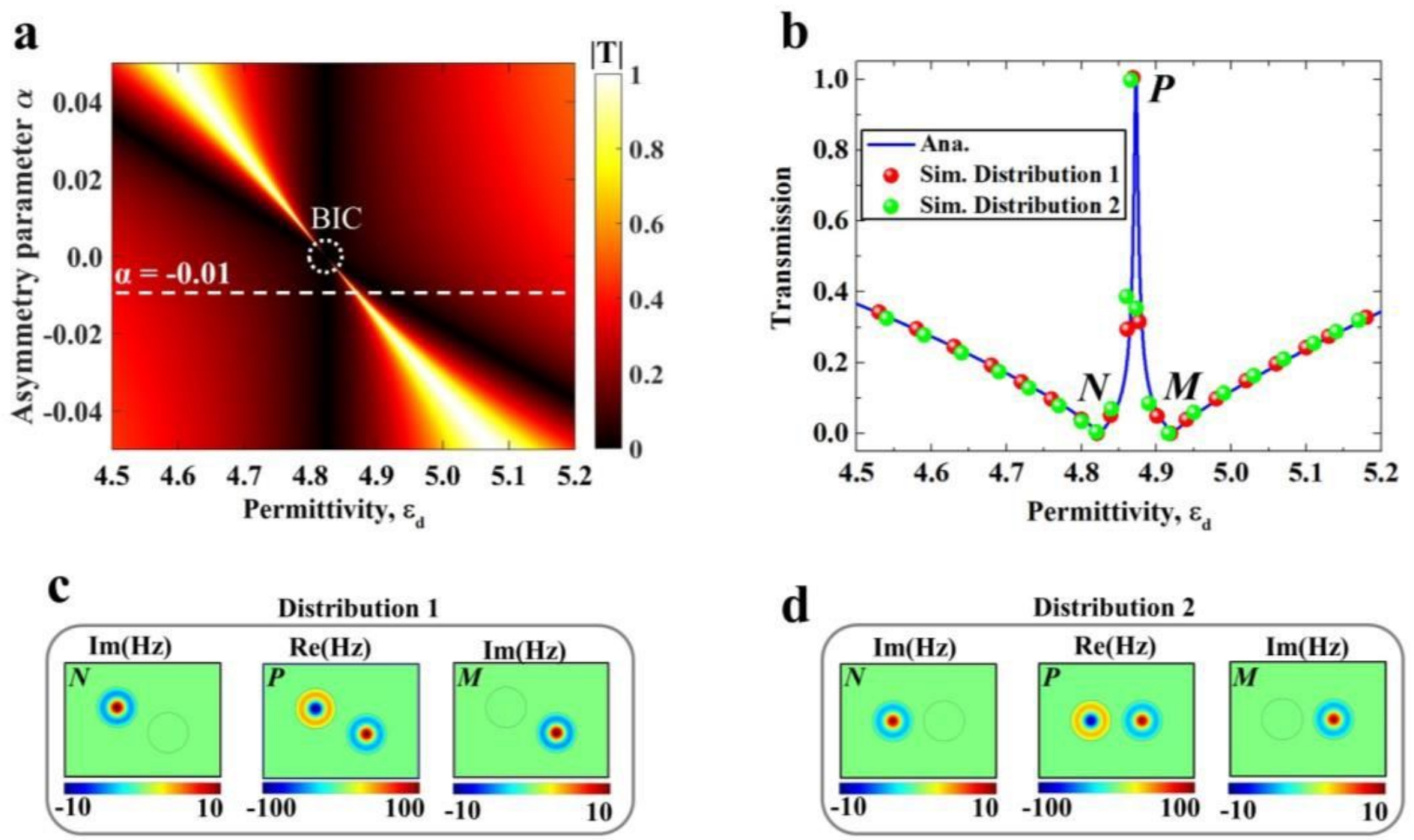

\section{Figure 2}

BIC illustrations. (see Manuscript file for full figure legend)

a

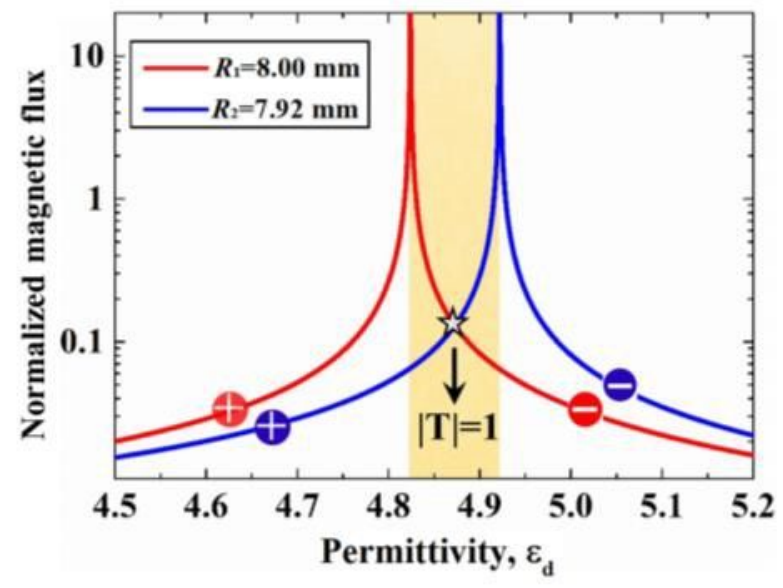

b

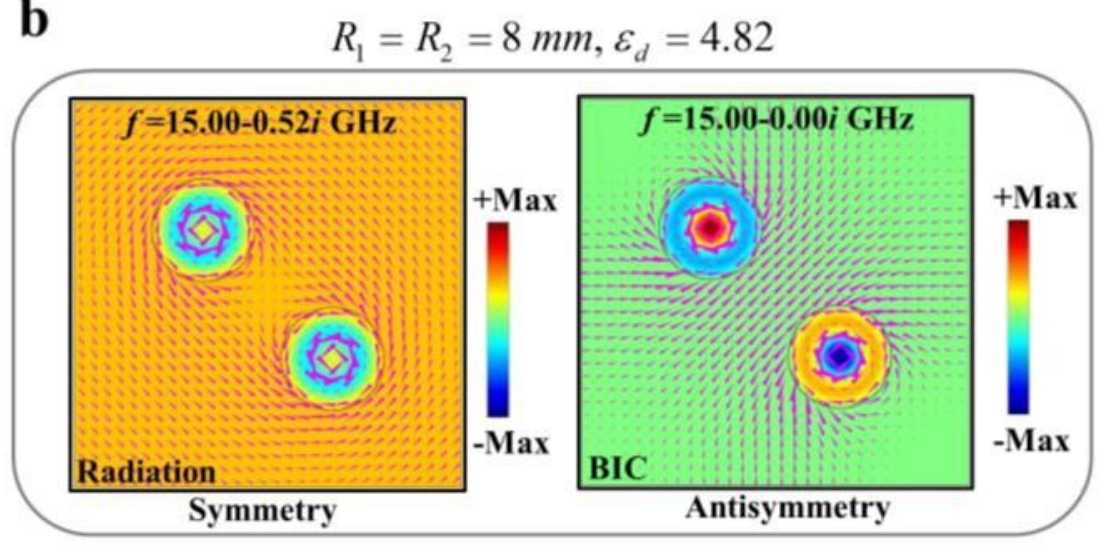

Figure 3

Normalized magnetic flux and eigenmode analysis. (see Manuscript file for full figure legend) 

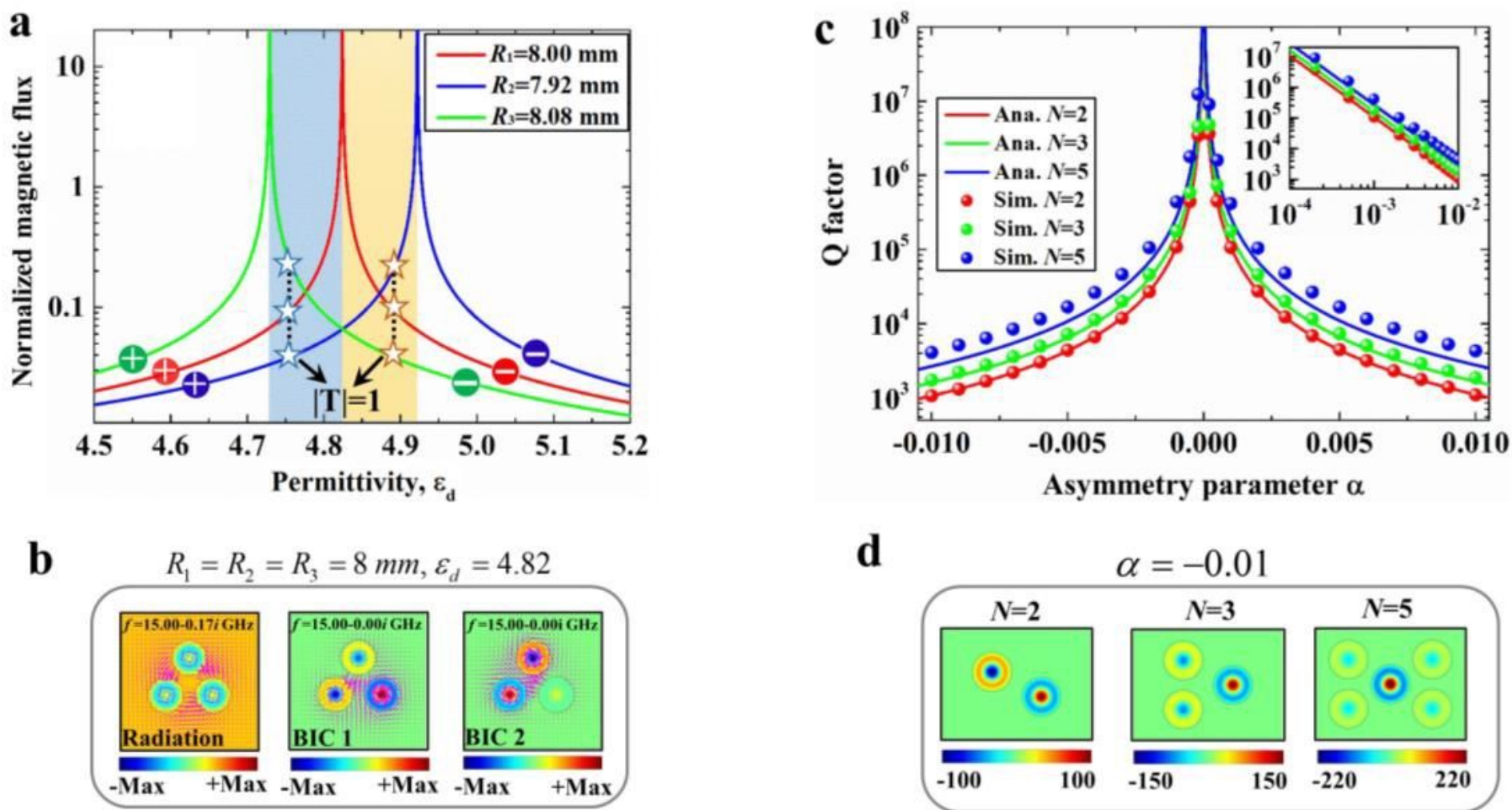

\section{Figure 4}

Higher-order optical BICs. (see Manuscript file for full figure legend) 

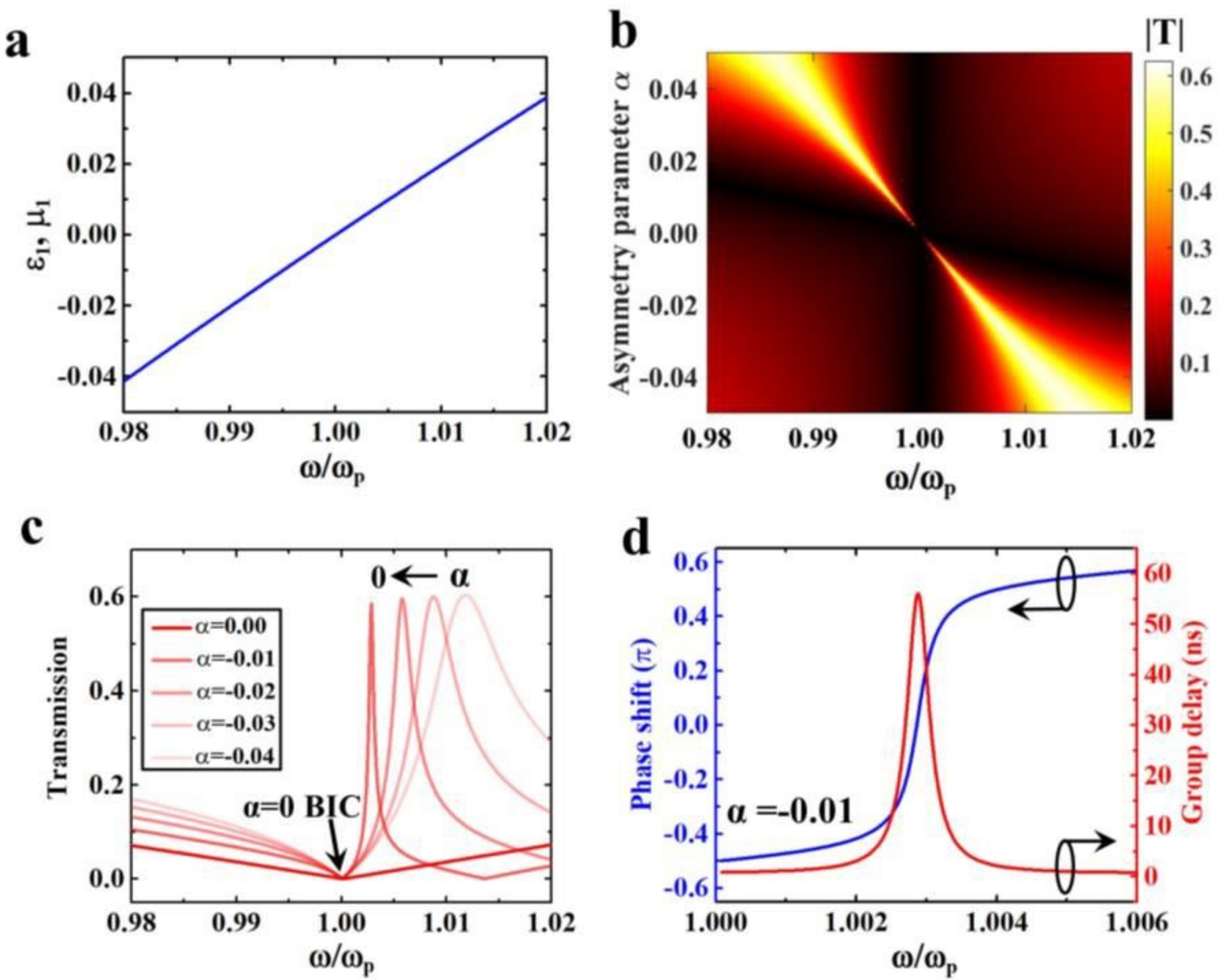

Figure 5

BIC frequency response in a dispersive ZIM with N=2 objects. (see Manuscript file for full figure legend) 
a

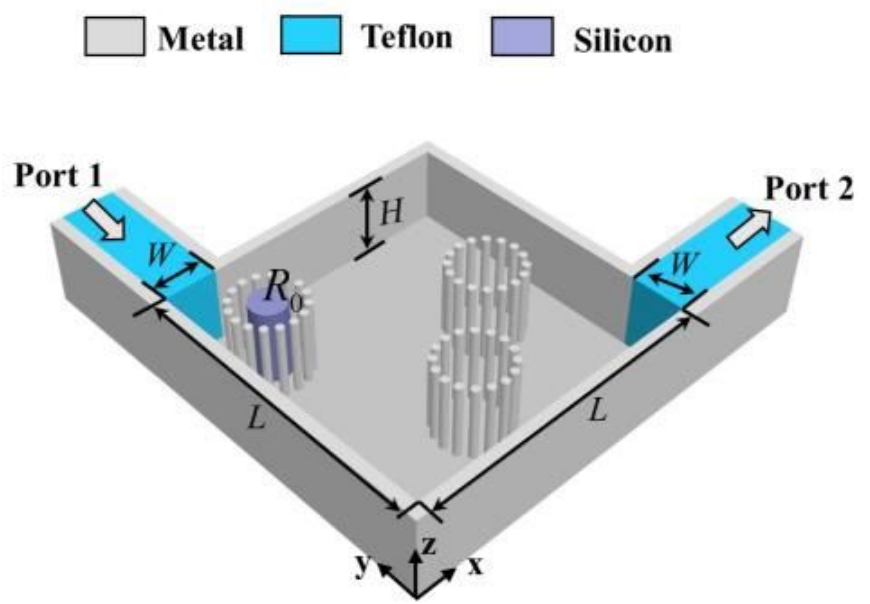

C

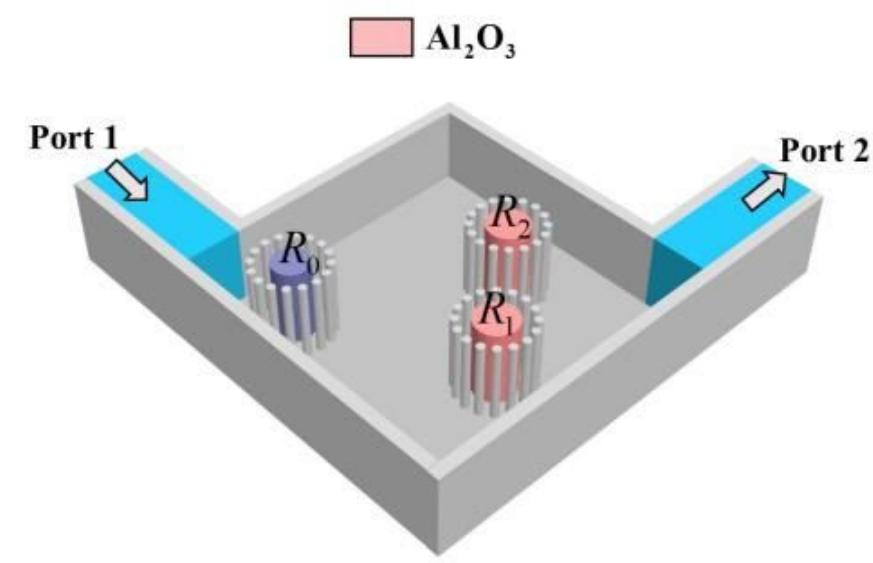

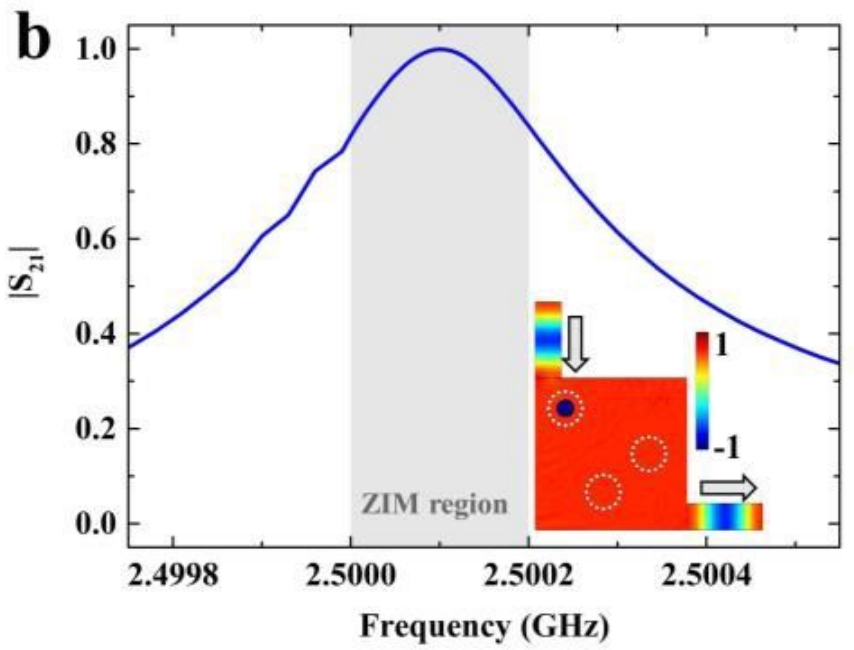

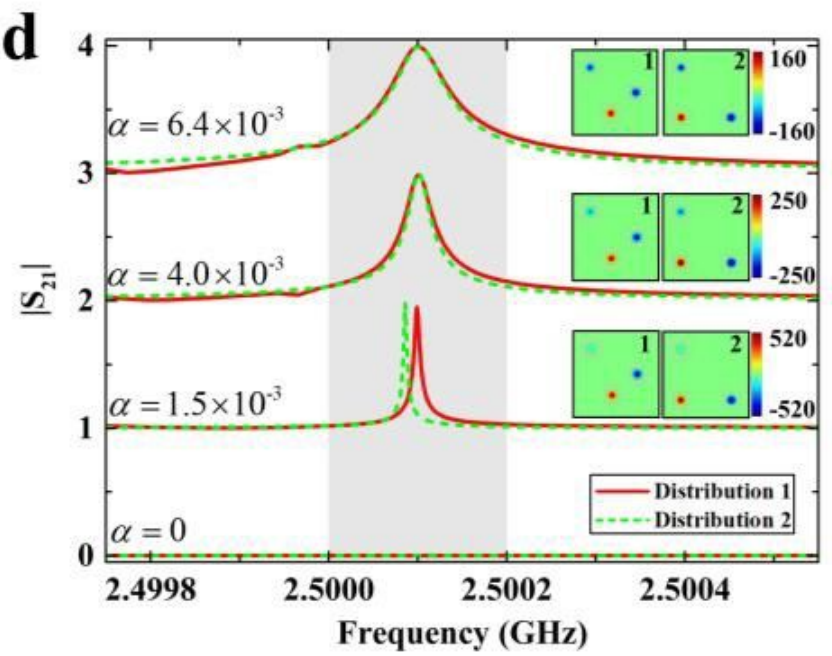

\section{Figure 6}

Experimental design for ZIM-induced BIC in a metallic rectangular waveguide system. (see Manuscript file for full figure legend)

\section{Supplementary Files}

This is a list of supplementary files associated with this preprint. Click to download.

- Supplementaryinformation1229.pdf 